\title{
Fundamentos metodológicos de la construcción de perfiles docentes universitarios
}

Methodological fundament of the construction of university teaching profiles

\author{
Soledad Vercellino \\ svercellino@unrn.edu.ar \\ Universidad Nacional de Río Negro, Centro \\ Interdisciplinario de Estudios Sobre Derechos, \\ Inclusión y Sociedad, Argentina

\section{Tatiana Gibelli} \\ tgibelli@unrn.edu.ar \\ Universidad Nacional de Río Negro, Centro \\ Interdisciplinario de Estudios Sobre Derechos, \\ Inclusión y Sociedad, Argentina

\section{Eduardo Lozano \\ elozano@unrn.edu.ar \\ Universidad Nacional de Río Negro, Centro de \\ Estudios e Investigación en Educación, Argentina}

Recepción: 23 Marzo 2021

Aprobación: 31 Mayo 2021

Publicación: 01 Junio 2021

Cita sugerida: Vercellino, S., Gibelli, T. y Lozano, E. (2021). Fundamentos metodológicos de la construcción de perfiles docentes universitarios. Revista Latinoamericana de Metodología de las Ciencias Sociales, 11(1), e087. https://doi.org/10.24215/18537863e087

\begin{abstract}
Resumen: En el marco de un proyecto de investigación, se analizan las trayectorias de formación de quienes cursan la Especialización en Docencia Universitaria en la Universidad Nacional de Río Negro. Un primer estudio se aboca a caracterizar, a través de la construcción de perfiles, a los docentes inscriptos en la primera cohorte en 2019. Este artículo propone describir el trabajo teórico, metodológico y operativo que implicó la conformación de estos perfiles. Inicialmente se analiza el uso de la noción de perfil como estrategia de ordenamiento de los datos. Luego se explicitan los procesos clasificatorios utilizados, que dieron lugar a los cinco perfiles identificados, brindando detalles sobre usos posteriores en la investigación. Como conclusión, se reflexiona sobre la utilidad de estas estrategias de investigación para abordar la multidimensionalidad del objeto de investigación.
\end{abstract}

Palabras clave: Perfil, Fundamentos metodológicos, Clasificación, Docencia universitaria.

\begin{abstract}
In the framework of a research project, the training trajectories of those who study the Specialization in University Teaching at the National University of Río Negro are analyzed. A first study focuses on characterizing the teachers enrolled in the first cohort in 2019, through the construction of profiles. This article proposes to describe the theoretical, methodological and operational work that involved the formation of these profiles. Initially, the use of the notion of profile as a data ordering strategy is analyzed. Then the classification processes used are made explicit, which gave rise to the five profiles identified, providing details on subsequent uses in the research. As a conclusion, it is reflected on the usefulness of these research strategies to address the multidimensionality of the research object.
\end{abstract}

Keywords: Profile, Methodogical fundament, University, Teaching. 


\section{INTRODUCCIÓN ${ }^{1}$}

Durante el año 2019, la Universidad Nacional de Río Negro (UNRN) inició el dictado de una Especialización en Docencia Universitaria (EDU). Esta iniciativa tuvo como objetivo atender a la formación para la enseñanza de docentes de la institución que son profesionales y, en su gran mayoría, no han cursado ni acreditado trayectorias de formación pedagógico-didáctica. En este sentido, la propuesta se enmarca en un modelo de desarrollo profesional del docente universitario con una doble vía: la profesión de origen en un campo específico disciplinario y la profesión en el campo de la docencia universitaria.

En ese marco, y de manera sincrónica con el dictado de la EDU, se implementó un proyecto de investigación denominado "Trayectorias de Formación en la Especialización en Docencia Universitaria de la Universidad Nacional de Río Negro”, acreditado por la UNRN (PI 40-A-719). Esta investigación se propuso analizar las trayectorias de formación de los profesores y profesoras que cursan la primera cohorte de esa Especialización (Lozano, Gibelli, Garelik, Vercellino y Ferrarino, 2020) para responder, entre otros, a estos interrogantes iniciales. ¿Qué perfiles de docentes de la UNRN que la cursan pueden configurarse? ¿Qué motivaciones los llevaron a cursar la EDU? ¿Qué sentidos y valoraciones les van otorgando a las instancias de formación que reciben y de qué manera advierten que se implican en resignificaciones de sus prácticas? A fin de responder a esas preguntas, se organizaron diferentes estudios. El primero de ellos consistió en caracterizar a los docentes inscriptos en la EDU y construir esos perfiles.

Este artículo tiene como objetivo describir, fundamentar y reflexionar sobre el trabajo teórico, metodológico y operativo que implicó conformar perfiles de docentes cursantes de la EDU. Para ello y, en primer lugar, analizaremos el uso de la noción de perfil como estrategia teórica metodológica de ordenamiento de los datos.

Luego explicitaremos los procesos clasificatorios utilizados para la conformación de perfiles. Caracterizaremos las tres operaciones clasificatorias implicadas en esa construcción: una intensiva, originada en los desarrollos teóricos en torno a las trayectorias profesionales docentes; otra extensiva, que surge de las similitudes en algunas características o propiedades comunes a ciertos docentes, y una orientada por los intereses del equipo de investigación. Finalmente, daremos cuenta de cómo esos perfiles fueron utilizados a lo largo de la investigación.

\section{SOBRE LA NOCIÓN DE PERFIL}

"Perfil" es una palabra que procede del provenzal antiguo, en el que se se refiere al borde de un vestido o dobladillo; su raíz latina "filare" significa "hilar" (Meza y Tobón, 2017). Según la RAE (2020), se asocia con la línea que marca el borde, contorno o figura de algo o alguien, que permite identificarlo y, por extensión, definir sus rasgos característicos.

El uso de la noción de perfil en el campo de las ciencias sociales en general, y las ciencias de la educación en particular, amerita un estudio en sí mismo. Se usa en términos prescriptivos o normativos para referirse a un conjunto de requisitos, competencias, tareas o funciones supuestas a diferentes unidades (por ejemplo: perfil del estudiante, del docente, del director, de un cargo). También se utiliza con funciones descriptivas, como noción que resume una diversidad de características, una multiplicidad de situaciones, fenómenos o individuos que comparten algún carácter más evidente o notorio y que puede identificarse como modelo, arquetipo, ejemplar, etc. Así se suele emplear de forma habitual en el lenguaje cotidiano y también en el lenguaje científico, coincidiendo con otras nociones también muy difundidas como las de tipo, clase, taxonomía. La distinción epistemológica y metodológica entre estos tres últimos conceptos es objeto de diferentes trabajos (Pérez Roldán, 1996; Marradi, 2000, 2007). Esta explicitación conceptual y operativa no ocurre con la noción de perfil, la que se utiliza sin mayores precisiones. 
En nuestra investigación la noción de perfil operó, en primer lugar, como instrumento de ordenamiento y clasificación de nuestras unidades de información, es decir, como herramienta descriptiva. El papel principal de las operaciones de clasificación en la producción de conocimiento ha sido reconocido como central en todo el desarrollo del pensamiento occidental desde la época de Aristóteles, y no ha estado exento de los riesgos del esencialismo. A esta cuestión le dedica un interesante ensayo Marradi (2000, 2007). El citado autor distingue tres categorías de operaciones clasificatorias que pueden dan lugar a diferentes resultados o productos: cuando se considera una subdivisión o una extensión de un solo concepto, el producto obtenido es una lista de clases, un esquema clasificatorio o simplemente una clasificación compuesta de diversas categorías llamadas "clases"; cuando se opera a la vez con diversos principios clasificatorios tratados simultáneamente y se obtiene una tipología con diversos tipos; y, por último, cuando estos principios clasificatorios se consideran como criterios sucesivos y jerárquicos, el orden clasificatorio resultante de la operación es una taxonomía que incluye diversos "taxa" (Marradi, 2000, 2007).

Como veremos más adelante, en el caso de los perfiles construidos se conformaron "clases"; es decir, agrupamientos de unidades de información según un único principio clasificatorio.

Asimismo, Marradi $(2000,2007)$ indica diferentes vías para las operaciones clasificatorias: una, cuya fuente es la teoría, que denomina "clasificación intensiva", en la que la extensión de un concepto ("genus") se subdivide en dos o más extensiones ("species") en un nivel menor de generalidad, de acuerdo con uno o varios criterios de división ("fundamentum" o "fundamenta divisiones"). Es una operación básica de elaboración conceptual en la que la intensión del concepto se articula y se clarifica en sus extensiones antes de ser reconocidas en la realidad empírica.

Otra operación es la que denomina "clasificación extensiva", la cual tiene como fuente principal la empiria. Aquí los objetos o fenómenos de un conjunto dado se agrupan en dos o más subconjuntos según las similitudes derivadas de una o varias propiedades. Hasta que los grupos son formados por algún procedimiento, no se establece el concepto que unifica cada combinación particular de elementos en el grupo constituido. En este procedimiento se recurre al uso de la estadística y no son pocos los trabajos que evalúan cuáles son los procedimientos y herramientas más adecuados en esos procesos de clasificación estadística (esquemas univariados, tablas de contingencia, esquemas multivariados, análisis clúster, etc.). Por ejemplo, el análisis de conglomerados o clústeres consiste en una técnica multivariante que busca agrupar elementos tratando de lograr la máxima homogeneidad en cada grupo y la mayor diferencia entre ellos. En este caso el investigador no tiene conocimiento de la existencia de los subgrupos o conglomerados de las características que los definen; es, por lo tanto, una técnica eminentemente exploratoria y descriptiva (Vilà-Baños, RubioHurtado, Berlanga-Silvente y Torrado-Fonseca, 2014).

En esta operación clasificatoria, la empiria es la fuente principal pero no exclusiva, pues la definición de las propiedades a partir de las cuales se conforman los grupos, su medición, suponen un conjunto de definiciones teóricas y metodológicas que se expresan en los instrumentos utilizados para relevar esos datos.

Por último, el tercer tipo de operación clasificatoria es identificada por el acto de asignar los objetos/ fenómenos a las distintas categorías que previamente han sido establecidas. Por tanto, esta operación es posterior a la definición de las categorías que se establecen a través tanto de la primera como de la segunda operación clasificatoria. Esta operación, además, puede aplicarse a objetos / fenómenos adicionales que no pertenecen al conjunto original que ha servido de base de la operación clasificatoria.

Como describiremos a continuación, en la construcción y conformación de perfiles de docentes de la UNRN que cursan la EDU recurrimos a esos tres tipos de operaciones descriptas por Marradi (2000, 2007).

\section{ELABORACIÓN DE PERFILES A PARTIR DE UNA "CLASIFICACIÓN INTENSIVA"}

La primera tarea que debimos darnos fue definir, desde algunas orientaciones teóricas del campo de la investigación educativa, qué clases de docentes cursantes de la EDU era posible y conveniente construir. 
En el marco de esa tarea, decidimos construir perfiles en función de sus trayectorias profesionales docentes. Entendemos las trayectorias profesionales como "itinerarios complejos, de ningún modo lineales", ocupadas en espacios que se transforman y que no pueden ser simplificadas a una serie de hitos o acreditaciones en un currículum (Bourdieu, 1997; Nicastro y Greco 2009; Lozano, Ruiz y Carranza, 2013).

Dicha complejidad es destacada por Feixas i Condom (2004), quien sostiene que la trayectoria docente del profesor universitario está influida por procesos significativos de tipo personales, familiares, institucionales (políticas de promoción, selección, formación y evaluación, recursos), contextuales (características de sus estudiantes y de los planes de estudios) y sociales (cambios tecnológicos y culturales). Coincide con Burke (1987), para quien existen dos grandes dimensiones que configuran la trayectoria profesional: la personal (las etapas vitales, las relaciones familiares, los incidentes críticos positivos, las crisis, las disposiciones individuales y las salidas no vocacionales) y la organizativa (las regulaciones de la profesión, los estilos de gestión, la administración pública, las expectativas sociales, las organizaciones profesionales y los sindicatos).

Los estudios sobre trayectorias profesionales de los docentes de nivel superior enfatizan la dimensión temporal de tales trayectos (Bolívar, Domingo y Fernández, 2001; Sánchez Olavarría y Huchim Aguilar, 2015; Fernández Cruz, 2008; Marquina, Yuni, y Ferreiro, 2017). Bolívar, Domingo y Fernández (2001) señalan que es común encontrar informes de investigación que refieran a los períodos por los que transita un docente durante su vida laboral en términos de fases de la vida, tiempos, ciclos de vida, cursos, etapas, estadios, estaciones, recorridos, carrera, trayectos, trayectorias o itinerarios.

Así, en función de esa variable temporal, Sánchez Olavarríay Huchim Aguilar (2015) clasifican a los docentes según su antigüedad en la docencia: docentes de trayectos iniciales, intermedios y avanzados; y según su continuidad en la docencia universitaria: docentes con trayectorias profesionales lineales, no lineales y discontinuas. Fernández Cruz (2008), por su parte, define trayectorias académicas: "tradicionaleshabituales", "prestigiosas acreditadas" y “combinadas". Finalmente, Marquina, Yuni, y Ferreiro (2017), con fuerte énfasis en la dimensión generacional, distinguen académicos consolidados, intermedios y noveles.

Se advierte en estas clasificaciones que toman como referencia una trayectoria que se desarrolla en el tiempo, de niveles de menor profesionalidad y prestigio a otros de acumulación de ambas cualidades, y que, además, suponen una trayectoria ideal, que a veces sufre accidentes o bifurcaciones.

A partir de este recorrido teórico, realizamos una clasificación intensiva de los y las docentes, tomando como "fundamenta divisiones" a la antigüedad en la docencia universitaria, y establecimos dos categorías diferenciales de docentes: por un lado, los docentes noveles, y por el otro, los docentes experimentados. Fueron considerados docentes noveles aquellos con menos de 5 años de ejercicio de la docencia y docentes experimentados los que acreditan más de 10 años. Al explorar esta variable en el total de docentes matriculados en la EDU se observó que el $76 \%$ tienen a lo sumo 10 años de ejercicio, es decir, la mayor proporción pertenece a la categoría de docentes noveles, mientras que el $24 \%$ restante conforma el grupo de docentes experimentados.

\section{Elaboración de PERFILES A PARTIR DE UNA CLASIFICACIÓN EXTENSIVA}

Ahora bien, junto a las dimensiones que surgen de la literatura, el equipo de investigación decidió jerarquizar otras dimensiones de las trayectorias profesionales que marcaran los perfiles docentes, pero construidas por otras vías: a partir de los resultados cuantitativos de una encuesta realizada como Estudio 1 del PI que caracterizó a los y las docentes.

El primer Estudio consistió en el desarrollo de una encuesta de carácter censal que permitió caracterizar a los-as ingresantes a la Especialización en Docencia Universitaria. La encuesta fue amplia en sus alcances: indagó en aspectos sociodemográficos de los estudiantes (Edad, Género, Nacionalidad, Localidad de nacimiento, Descendiente de pueblos originarios, Ciudad en la que reside en relación con la Sede en la que trabaja, Hijos, Tipo de vivienda); otros, vinculados a su trayectoria académica y profesional (Formación 
de grado / universidad-institución otorgante; Formación de posgrado / universidad-institución otorgante; Generación de universitarios en la familia, Años de antigüedad en la docencia, Trabajo no docente en ámbitos públicos o privados / años de antigüedad, Cargo docente / Dedicación / Condición; Área disciplinar, año de la carrera en el que se desempeña como docente, características de su participación en la vida universitaria, influencia de la investigación y la extensión, etc.). También se indagó sobre sus motivaciones para cursar la EDU.

Se implementaron 134 encuestas: 51 en Sede Atlántica (38 \%), 48 en Sede Alto Valle y Valle Medio (36 $\%)$ y 35 en Sede Andina (26\%).

Los agrupamientos de docentes, surgidos a partir del análisis de los datos recogidos en esta encuesta, surgen de considerar las similitudes derivadas a partir del análisis de frecuencias de varias características. En este procedimiento se recurrió al uso de la estadística descriptiva, sin avanzar en análisis multivariados, de conglomerados o clústeres, debido a la escasez de variables cuantitativas en el instrumento utilizado.

Del total de inscriptos, hay 94 que trabajan en UNRN, lo que representa el $70 \%$. El resto desarrolla tareas sólo en otras universidades. En relación con su dedicación horaria en la docencia universitaria, el 72 \% de los/as docentes posee dedicación simple.

Por otro lado, advertimos que entre los docentes que trabajan en la UNRN, 69 de ellos desarrollan además tareas profesionales en ámbitos no docentes (lo que representa el 73 \%). Asimismo, un $22 \%$ se desempeña como docente en otras instituciones de educación superior.

A partir del valor asignado a estos datos, se consideró pertinente construir una clase con aquellos docentes cuya principal actividad es el ejercicio profesional, con la docencia como una tarea secundaria en dedicación horaria, frente a aquellos cuya profesión es la académica, exclusivamente.

\section{Elaboración DE PERFILES A PARTIR DE CRITERIOS PRIORIZADOS POR EL EQUIPO DE INVESTIGACIÓN}

Finalmente, se propusieron dos perfiles docentes más, que no surgieron por criterio intensivo y tampoco exclusivamente extensivo, sino a partir de poner en valor ciertos datos provenientes del estudio de matrícula en el marco de temáticas y de intereses que se identifican en la propia vida institucional de la UNRN.

La Universidad Nacional de Río Negro (UNRN), creada en diciembre de 2007 por ley 26330, inició el dictado de clases en marzo de 2009, es decir, hace poco más de 10 años. Con una matrícula de 9962 estudiantes que desarrollan algún tipo de actividad (UNRN, 2020), a octubre del 2019 contaba con 1919 estudiantes que habían egresado (Oficina de Aseguramiento de la Calidad de la Universidad Nacional de Río Negro, 2020). Considerando que el Proyecto de Investigación se propone aportar conocimientos que sirvan para la mejora de la calidad institucional, se definió, como un perfil relevante de indagar, el de aquellos docentes que fueran "graduados" de esta institución, los que constituyen el $10 \%$ de la matrícula de la EDU.

Finalmente interesó, en el marco de las políticas institucionales, identificar a aquellos docentes que "enseñan en el primer año de las carreras", es decir, docentes vinculados a la problemática del inicio de los estudios universitarios. El problema del ingreso es un problema jerarquizado por la UNRN, la que, a lo largo de la última década, ha desarrollado diversos dispositivos, planes, programas con la intención de mejorar los resultados de aprendizaje e indicadores de rendimiento de ese período inicial de los estudios universitarios. Así, en línea con los objetivos establecidos en el Plan de Desarrollo Institucional (PDI) 2019/2025, el Consejo Superior de Docencia, Extensión y Vida Estudiantil de la UNRN ha aprobado en el mes de agosto de 2019 los "Lineamientos Institucionales de ingreso a la UNRN -cohorte 2020-", cuya acción central es la implementación de un curso de ingreso obligatorio e introductorio a la universidad y a la carrera (Vercellino, Gibelli, Misischia y Goin, 2019).

De la encuesta inicial, advertimos que el $42 \%$ de los estudiantes de la EDU dictan asignaturas en primer año y de este modo quedó definido el quinto perfil. 
En síntesis, a partir de criterios teóricos, estadísticos y de intencionalidad/ significatividad para el estudio se han definido cuatro criterios y seis perfiles/clases, según se muestra en la Tabla 1.

TABLA 1

Conformación de clases de docentes

\begin{tabular}{|c|c|}
\hline CRITERIOS & CLASES \\
\hline Antigüedad en la docencia universitaria & Noveles / Experimentados \\
\hline Dedicación horaria a la docencia universitaria & Profesionales / Académicos \\
\hline Lugar de graduación & En UNRN / En otras Universidades \\
\hline Año en el que dictan sus asignaturas & Primer año de carreras / Otros años de carreras \\
\hline
\end{tabular}

Los perfiles surgen de esas operaciones de clasificación, es decir, de generar agrupamientos de unidades de información según un único principio o criterio.

Luego, procedimos al tercer tipo de operación clasificatoria que describe Marradi (2000, 2007) y asignamos los casos a los distintos perfiles establecidos, para dar continuidad a los estudios cualitativos implicados en la investigación.

\section{VARIABILIDAD DENTRO DE LOS PERFILES}

La encuesta inicial permitió caracterizar los cargos y dedicaciones horarias de los docentes que cursan la EDU, y los resultados indican que el grueso de la población se compone de auxiliares de docencia. Un 38 \% son Jefes de Trabajos Prácticos, la mayoría de ellos a cargo de las asignaturas, y un $34 \%$ Ayudantes de Primera. El $25 \%$ restante son Profesores y el porcentaje de docentes interinos es del $56 \%$.

Por otra parte, la dispersión de formaciones disciplinares de base fue acotada a cinco campos: Salud, Ciencias Naturales y Exactas, Humanidades, Ingenieros y Técnicos y Ciencias Sociales (Ver Tabla 2).

TABLA 2

Formaciones disciplinares de los docentes que cursan la EDU en la UNRN

\begin{tabular}{|c|c|c|}
\hline \multicolumn{2}{|c|}{ Campo disciplinar } & Titulaciones \\
\hline \multicolumn{2}{|c|}{ Salud } & $\begin{array}{l}\text { Odontología. Bioquímica. Kinesiología y Fisiatría. Enfermería. Nutrición. } \\
\text { Terapia Física. Medicina Humana. Medicina Veterinaria }\end{array}$ \\
\hline \multicolumn{2}{|c|}{$\begin{array}{c}\text { Ciencias Exactas y } \\
\text { Naturales }\end{array}$} & Biología. Biotecnología. Matemática. Geología \\
\hline \multicolumn{2}{|c|}{ Humanidades } & Arquitectura. Cine. Inglés \\
\hline \multicolumn{2}{|c|}{ Ingenieros y Técnicos } & $\begin{array}{l}\text { Diseño Industrial. Ingeniería en: Sistemas, Civil. Electrónica, } \\
\text { Agronomía. Alimentos. Tecnología. Tecnicatura en Industria. Tecnología }\end{array}$ \\
\hline \multirow{3}{*}{$\begin{array}{l}\text { Ciencias } \\
\text { Sociales }\end{array}$} & Economía & $\begin{array}{l}\text { Administración de empresas. Contador Público. Administración. } \\
\text { Marketing. Producción }\end{array}$ \\
\hline & Sociales & $\begin{array}{l}\text { Comunicación. Sociología. Turismo. Abogacía. Ciencias Políticas. } \\
\text { Criminología }\end{array}$ \\
\hline & Educación & $\begin{array}{l}\text { Psicopedagogía. Educación. Profesorado de Educación Física, de } \\
\text { Educación Especial, Inglés, Biología, Física, Química, Comunicación }\end{array}$ \\
\hline
\end{tabular}


Estos aspectos, descriptos en el análisis de la matrícula inicial, no fueron considerados en el proceso de elaboración de los perfiles a partir de los criterios antes expuestos, sino que intencionadamente fueron utilizados para otorgar variabilidad (Berteaux, 2005) al conjunto de casos de docentes seleccionados para cada perfil docente.

\section{USOS DE LAS CLASIFICACIONES EN LA INVESTIGACIÓN}

La conformación de estos perfiles resultó un ordenador del proceso de muestreo para los estudios subsiguientes, particularmente el tercer estudio del PI, el que busca a partir de entrevistas semiestructuradas profundizar la compresión de las trayectorias de formación que se llevan a cabo en la EDU, describiendo los sentidos y las implicancias en la tarea docente que los cursantes atribuyen a dicho espacio de formación.

Así, para el Estudio $\mathrm{N}^{\circ} 3$ se conformó una muestra de docentes estudiantes de la EDU por Sede. La muestra supuso la selección de tres docentes dentro de cada perfil/clase. No se consideraron todos los perfiles sino cinco: 1. docentes noveles; 2. docentes experimentados; 3. docente cuya principal actividad es la profesional/ disciplinar; 4. docentes graduados de la UNRN y 5. docentes de primer año. De este modo, la muestra teórica quedó conformada por nueve casos para cada perfil, la que luego se vio modificada por restricciones en su implementación (docentes que no aceptaron o diferencias cuantitativas entre sedes, por ejemplo, en cuanto a la distribución de casos para el perfil "graduados de la UNRN", entre otras)

El criterio de variabilidad al interior de cada perfil se dio, como ya se ha adelantado, teniendo en cuenta las áreas de conocimiento de la disciplina de base y la dedicación a la docencia. Ante casos iguales se recurrió al azar. La Tabla 3 es una tabla de contingencia o de múltiples entradas que da cuenta del esquema clasificatorio utilizado y del nivel de variabilidad alcanzado. 
TABLA 3

Proceso de selección de casos para la Sede Atlántica. En resaltado, los casos seleccionados

\begin{tabular}{|c|c|c|c|c|c|c|c|}
\hline \multirow[b]{2}{*}{ PERFIL } & \multirow[b]{2}{*}{$\begin{array}{l}\mathrm{N}^{0} \\
\text { iden. }\end{array}$} & \multicolumn{5}{|c|}{ TITULACIÓN } & \multirow[b]{2}{*}{$\begin{array}{l}\text { DEDICA- } \\
\text { CIÓN }\end{array}$} \\
\hline & & Salud & $\begin{array}{l}\text { Exactas y } \\
\text { Naturales }\end{array}$ & $\begin{array}{c}\text { Humani- } \\
\text { dades }\end{array}$ & $\begin{array}{l}\text { Ingenieros } \\
\text { y técnicos }\end{array}$ & Sociales & \\
\hline \multirow{9}{*}{$\begin{array}{l}\text { 1. Docentes } \\
\text { Noveles }\end{array}$} & 8 & & & & & $\mathrm{X}$ & simple \\
\hline & 27 & $\bar{X}$ & & & & & simple \\
\hline & 29 & $\bar{X}$ & & & & & simple \\
\hline & 32 & $\bar{X}$ & & & & & simple \\
\hline & 39 & & & & $\mathrm{X}$ & & simple \\
\hline & 43 & $\mathrm{X}$ & & & & & simple \\
\hline & 44 & & & & & $\mathrm{X}$ & simple \\
\hline & 47 & & & & & $\bar{X}$ & simple \\
\hline & 49 & & & & & $\mathrm{X}$ & $\mathrm{n} / \mathrm{s}$ \\
\hline \multirow{5}{*}{$\begin{array}{l}\text { 2. Docentes } \\
\text { experimentados }\end{array}$} & 5 & & & & & $\bar{X}$ & completa \\
\hline & 6 & & & & X & & completa \\
\hline & 15 & & & & $\mathrm{X}$ & & completa \\
\hline & 45 & & $\mathrm{X}$ & & & & completa \\
\hline & 46 & & $\mathrm{X}$ & & & & completa \\
\hline \multirow{6}{*}{$\begin{array}{l}\text { 3. Docentes } \\
\text { que desarrollan } \\
\text { trabajo } \\
\text { profesional }\end{array}$} & 2 & & & & & $\bar{X}$ & simple \\
\hline & 11 & $\mathrm{X}$ & & & & & parcial \\
\hline & 20 & & & & & $\bar{X}$ & simple \\
\hline & 24 & & & & & $\bar{X}$ & simple \\
\hline & $26 \mathrm{~A}$ & & & & & $\bar{X}$ & simple \\
\hline & $26 \mathrm{~B}$ & $\bar{X}$ & & & & & simple \\
\hline \multirow{2}{*}{$\begin{array}{l}\text { 4. Egresados } \\
\text { UNRN }\end{array}$} & 10 & & & & & $\bar{X}$ & simple \\
\hline & 41 & & & & & $X$ & simple \\
\hline \multirow{11}{*}{$\begin{array}{l}\text { 5. Docentes } \\
\text { que trabajan en } \\
\text { 1er año }\end{array}$} & 1 & $\mathrm{X}$ & & & & & parcial \\
\hline & 3 & & & & & $\mathrm{X}$ & simple \\
\hline & 6 & & & & $\mathrm{X}$ & & completa \\
\hline & 14 & $\bar{X}$ & & & & & simple \\
\hline & 16 & & & & $\bar{X}$ & & simple \\
\hline & 21 & & & & & $\mathrm{X}$ & simple \\
\hline & 23 & & & & & $\mathrm{X}$ & simple \\
\hline & 31 & $\mathrm{X}$ & & & & & simple \\
\hline & 37 & $\mathrm{X}$ & & & & & simple \\
\hline & 40 & $\mathrm{X}$ & & & & & simple \\
\hline & 48 & $\mathrm{X}$ & & & & & simple \\
\hline
\end{tabular}

La primera estrategia cualitativa de recolección de datos consistió en la implementación de una entrevista semiestructurada a los casos seleccionados. ${ }^{2}$ Se organizó en dos bloques de preguntas: uno común a todos los estudiantes de la EDU y otro por perfil.

En el Bloque Común se indagó sobre la biografía profesional (cómo y cuándo se decidió por la docencia universitaria, cómo fue la trayectoria previa que llevó al ingreso a la UNRN y cómo fue el devenir de su trayectoria hasta la actualidad); las motivaciones para realizar la EDU, su valoración de ella y el impacto/ influencia de ésta en diferentes aspectos de su tarea docente.

El bloque específico supuso una serie de interrogantes para cada perfil definido, como se advierte en la Tabla 4, en la que se indican los aspectos especialmente indagados para cada perfil. 
TABLA 4

Aspectos indagados en entrevista, según perfil

\begin{tabular}{|c|c|}
\hline PERFIL & ASPECTOS INDAGADOS \\
\hline $\begin{array}{l}\text { DOCENTES } \\
\text { NOVELES }\end{array}$ & $\begin{array}{l}\text { Valoración sobre el proceso de formación como docente universitario } \\
\text { Dificultades/desafios }\end{array}$ \\
\hline $\begin{array}{l}\text { DOCENTES } \\
\text { EXPERIMENTADOS }\end{array}$ & $\begin{array}{l}\text { Problemas persistentes en la docencia universitaria. Nuevos } \\
\text { problemas } \\
\text { Cambios en las prácticas de enseñanza } \\
\text { Situaciones significativas que impactan en la actividad docente } \\
\text { La formación actual de los docentes }\end{array}$ \\
\hline $\begin{array}{l}\text { DOCENTE CON } \\
\text { ACTIVIDAD } \\
\text { PROFESIONAL }\end{array}$ & $\begin{array}{l}\text { Diferencias entre la práctica profesional y la práctica docente } \\
\text { Vinculaciones entre ambas prácticas y ámbitos laborales }\end{array}$ \\
\hline $\begin{array}{l}\text { DOCENTES } \\
\text { GRADUADOS DE LA } \\
\text { UNRN }\end{array}$ & $\begin{array}{l}\text { Consideraciones sobre la continuidad de la formación de } \\
\text { grado/ingreso a la docencia/ formación en la EDU } \\
\text { Valoración de su formación de grado } \\
\text { Impacto de la EDU en la valoración de su formación de grado y de } \\
\text { las prácticas docentes }\end{array}$ \\
\hline $\begin{array}{l}\text { DOCENTES DE } \\
\text { PRIMER ANNO }\end{array}$ & $\begin{array}{l}\text { Razones por las que enseña en primer año } \\
\text { Motivos de la no continuidad de Ixs estudiantes y si la EDU ha hecho } \\
\text { modificar sus percepciones al respecto } \\
\text { Su visión sobre cómo se enseña en primer año } \\
\text { Dificultades para la enseñanza en ese año } \\
\text { Estrategias desarrolladas } \\
\text { Participación en reuniones institucionales/ acuerdos }\end{array}$ \\
\hline
\end{tabular}

\section{Conclusiones}

A lo largo de este trabajo hemos descripto y fundamentado el proceso teórico y metodológico de construcción de perfiles docentes. Intentamos reconstruir y fundamentar una de las operaciones que en la investigación nos permitió abordar la multidimensionalidad de nuestro objeto (las vicisitudes en las trayectorias de formación de docentes universitarios y su vinculación con los sentidos que dan a su práctica docente en el marco de la EDU). Se trata de dar cuenta de algunas de las estrategias de investigación necesarias para que dicha multidimensionalidad logre reducirse y hacerse, en alguna medida, comprensible.

Las operaciones de clasificación y producción de perfiles consisten - como no deja de insistir la literatura en la materia- en herramientas que sirven para ponderar o destacar aspectos de la realidad social descrita, mientras que dejan en la sombra otros aspectos considerados como menos relevantes. No dicen la realidad sino que son los instrumentos utilizados para analizarla, una heurística para "dar coherencia narrativa a la abrumadora y caótica evidencia de la experiencia humana” (Bauman, 2007, citado en Cohen y Rojas, 2011, p. 43)

Los perfiles resultaron del producto de tres operaciones clasificatorias: una intensiva, originada en los desarrollos teóricos en torno a las trayectorias profesionales docentes; otra extensiva, que surge de las similitudes en la frecuencia de algunas características o propiedades comunes a ciertos docentes, y una orientada por los intereses del equipo de investigación. Luego se asignaron los docentes a las distintas categorías que previamente habían sido establecidas. 
Los criterios de ordenamiento adquieren en el proceso de clasificación un rol fundamental, dado que su elección e inclusión determinan las características y significación que asumen los grupos resultantes. Así, a partir de esas operaciones clasificatorias y de diferentes criterios (antigüedad en la docencia universitaria, dedicación horaria a la docencia universitaria, lugar de graduación, año en el que dictan sus asignaturas), se han definido cinco perfiles docentes: 1 . docentes noveles; 2 . docentes experimentados; 3 . docentes cuya principal actividad es la profesional/disciplinar; 4. docentes graduados de la UNRN y 5. docentes de primer año. No se trata de una clasificación exclusiva y excluyente, sino de la jerarquización de ciertas peculiaridades, propiedades o notas distintivas que resultan significativas para la investigación y que permite conglomerar a ciertos docentes que las comparten, y comparar los sentidos y significados que ellos producen con los que construyen otros y otras docentes que soportan otros rasgos o características.

Asimismo, la definición de ciertas características transversales como las áreas de conocimiento de la disciplina de base y la dedicación a la docencia ha permitido, dentro de cada perfil, incorporar cierta variabilidad.

La reducción del espacio de propiedades y dimensiones que posee un fenómeno social, como las trayectorias profesionales docentes, a unos pocos criterios de clasificación, implica tomar una serie de decisiones teóricas sobre el modo en que pensamos la relación entre las distintas dimensiones y el objeto que finalmente buscamos construir. Los criterios de referencia no son neutros: se asientan en una serie de conjeturas que el equipo de investigación sostiene, originada tanto en la literatura sobre la materia como en la problematización de los procesos sociales que intentan explicar. Vale aclarar, entonces, que el peso diferencial otorgado a ciertas dimensiones influye decisivamente en la estimación de la significación de los perfiles resultantes.

El desarrollo de los estudios subsiguientes nos permitirá validar esta construcción de perfiles. Eso será objeto de otra comunicación.

\section{ReFERENCIAS}

Berteaux, D. (2005). Los relatos de vida. Perspectiva etnosociológica. Barcelona: Ediciones Bellaterra.

Bolívar, A., Domingo, J. y Fernández Cruz, M. (2001). La investigación (auto) biográfica narrativa. Guía para la indagación en el campo. Granada: . Force.

Bourdieu, P. (1997). Razones prácticas. Sobre la teoria de la acción. Barcelona: Anagrama.

Burke, P. J. (1987). Teacher Development. Induction, Renewal and Redirection. Londres: The Falmer Press.

Cohen, N. y Rojas, G. G. (2011). Las tipologías y sus aportes a las teorías y la producción de datos. Revista Latinoamericana de Metodología de la Investigación Social: ReLMIS, 1, 36-46.

Feixas i Condom, M. (2004). La influencia de factores personales, institucionales y contextuales en la trayectoria y el desarrollo docente de los profesores universitarios. Educar, 33, 31-59.

Fernández Cruz, M. (2008). El prácticum en la construcción del conocimiento profesional docente. En: Correa, E. (coord.). Vers une conceptualisation de la situation de stage: explorations internationales. Sherbrooke: Éditions CRP.

Lozano, E., Ruiz, M. E. y Carranza, P. (2013). Trayectorias personales e identidades profesionales: "Ingenieros y Licenciados copan el nivel medio". Cuadernos de Educación, XI(11), 1-13. Recuperado de https://rid.unrn.ed u.ar/jspui/handle/20.500.12049/3478

Lozano, E. E., Gibelli, T., Garelik, C. E., Vercellino, S., y Ferrarino, C. (2020). Trayectorias de formación en una especialización en docencia universitaria. III Jornadas sobre las prácticas docentes en la Universidad Pública. Universidad Nacional de La Plata. (Edición en línea, junio de 2020). Recuperado de https://rid.unrn.edu.ar/h andle/20.500.12049/4911. 
Marquina, M., Yuni, J. A., y Ferreiro, M. (2017). Trayectorias académicas de grupos generacionales y contexto político en Argentina: Hacia una tipología. Education Policy Analysis Archives/Archivos Analiticos de Politicas Educativas, $25,1-23$.

Marradi, A. (2000). Reivindicando el papel de la tipología: de Aristóteles a las Ciencias Humanas Modernas. Nómadas: Critical Journal of Social and Juridical Sciences, 1, 15.

Marradi, A. (2007). La tipología desde Aristóteles a las ciencias sociales modernas. Revista de Ciencia Politica y de Relaciones Internacionales (en línea), 1(1), 4-24.

Nicastro, S., y Greco, M. B. (2009). Entre trayectorias. Escenas y pensamientos en espacios de formación. Rosario: Homo sapiens.

Oficina de Aseguramiento de la Calidad de la Universidad Nacional de Río Negro (2020). Los egresados de la UNRN. Viedma. Recuperado de https://rid.unrn.edu.ar/jspui/handle/20.500.12049/4337

Pérez Roldán, L. (1996). La construcción de tipologías: metodología de análisis. Papers. Revista de Sociología, 48, 9-29.

Sánchez Olavarría, C., y Huchim Aguilar, D. (2015). Trayectorias docentes y desarrollo profesional en el nivel medio superior. Revista CPU-e, (21), 148-167.

Universidad Nacional de Río Negro (2020). Memoria institucional 2019. Viedma: Universidad Nacional de Río Negro.

Vercellino, S., Gibelli, T., Misischia, B. y Goin, M. (2019). Ingresos e ingresantes: hacia una comprensión multidimensional de los procesos institucionales y subjetivos implicados en los inicios de los estudios universitarios. Proyecto de Investigación presentado ante la Universidad Nacional de Río Negro. Viedma, mímeo.

Vilà-Baños, R., Rubio-Hurtado, M. J., Berlanga-Silvente, V. y Torrado-Fonseca, M. (2014). Cómo aplicar un cluster jerárquico en SPSS. REIRE, Revista d'Innovació i Recerca en Educació, 7(1), 113-127. Recuperado de http://w ww.ub.edu/ice/reire.htm

\section{NoTAS}

1 Las opiniones y/o ideas expresadas no representan necesariamente las opiniones y/o posiciones de la Universidad Nacional de Río Negro.

2 Disponible en https://www.mediafire.com/file/x465glyf $2 \mathrm{k} 7 \mathrm{u} 2 \mathrm{kn} /$ Protocolo_Entrevista_semiestructurada_bloque_c om\%FAn_-_perfiles.pdf/file) 